\title{
RELACION DE TESIS Y TESINAS SOBRE HISTORIA DE ESPAÑA LEIDAS EN LA UNIVERSIDAD DE TOULOUSE-LE MIRAIL (1965-1985)
}

\author{
F. Brumont ${ }^{1}$
}

\author{
RESUMEN
}

\begin{abstract}
Se expone en esta breve colaboración una serie de títulos de tesis y tesinas leidas en la Universidad de Toulouse sobre historia de España, con un objetivo puramente bibliográfico y pensando en la ayuda que puede obtener el investigador español. La gran mayoría de ellas pueden ser consultadas en la casa de Velázquez o a través de esta institución.
\end{abstract}

\section{SUMMARY}

In this brief collaboration article a series of thesis and dissertation titles is given. They were read in the University of Toulouse and they all deal with spanish history.

Me propongo en este corto artículo dar a conocer a los estudiosos españoles la relación de los trabajos hechos por los alumnos de historia de la Universidad de Toulouse sobre historia de España en los últimos veinte años. Todos (menos el n. ${ }^{\circ}$ 27) se realizaron bajo la dirección del prof. B. BENNASSAR, ayudado en algunso casos por el prof. J. P. AMALRIC.

Casi todos son memorias de licenciatura (mémoires de maîtrise), siendo los demás, tesis que llamamos de Tercer Ciclo, que suelen hacerse en dos o tres años (en cuyo caso pongo tesis al final de la relación). No se hace un análisis del contenido de dichas obras ya que, de manera general, los títulos son bastante explícitos al respecto. En cambio, aparecen las fuentes utilizadas. Ta mbién se hace mención, en su caso, de la publicación de la obra o de trabajos realizados a partir de la misma. Cabe añadir que la mayoría de estos trabajos se pueden encontrar en la biblioteca de la Casa de Velázquez en Madrid, donde han sido depositados ejemplares fotocopiados; aparecen en la relación señalados con un asterisco.

Como cabía esperar la mayoría de ellos se refieren a la época moderna (son 37 de 42) mientras 4 son estudios de la España más contemporánea (de 1930 a 1976), no teniendo el

1. C.N.R.S. Universidad de Toulouse-Le Mirail. 
último límites cronológicos ya que trata de la visión de España por los viajeros extranjeros (n. ${ }^{\circ}$ 24). Si desglosamos por siglos, el vencedor sería el XVI (12 títulos) seguido del XVII (8) mientras 4 abarcan ambos siglos, el XVIII (7), el XX (4 como queda dicho) y el XIX, con 3 , todos referidos a la guerra de la Independencia.

En cuanto a las fuentes, los documentos más utilizados son los que proceden del archivo de la Inquisición y después los Expedientes de Hacienda del AGS; curiosamente, el Catastro de la Ensenada sólo ha sido utilizado dos veces.

Estos estudios cubren toda la geografía española: Aragón, Canarias y Murcia son las únicas regiones que no aparecen. La zona más estudiada es Andalucía ( 8 títulos) seguida de cerca por Castilla la Vieja (7), Valencia (6) y Castilla la Nueva (5). Un poco rezagados aparecen Extremadura (3), Asturias (2), Baleares, Santander, Galicia, Cataluña y La Rioja con uno (este último, n. ${ }^{\circ} 23$, estudia también el País Vasco).

Los temas más frecuentes son los relacionados con demografía, economía y sociedad (19 titulos) y los estudios de mentalidades (12); se puede mencionar a este respecto que éstos se desarrollan a partir de 1973 (curso 1972-73) desbancando a aquéllos que vuelven a aparecer estos últimos años.

P or fin, tengo que decir que en varios casos se prosiguen las investigaciones; así es que V. BLAZY está prosiguiendo su estudio sobre el cine, al estudiar la imagen de España en el cine mundial y que B. FERNANDEZ acaba su tesis de Tercer Ciclo sobre el districto inquisitorial de Logroño (el balance de sus trabajos anterior aparece en esta misma resvista).

A modo de conclusión, sólo decir que espero que este trabajo anime a otros departamentos y que se publiquen más listas de trabajos inéditos para el bien común de cuantos investigamos sobre temas españoles.

1. ALONSO, M. Le «Diario de Valencia» sous l'occupation française (février 1812 à juillet 1813). 1980. $170 \mathrm{p}$.

Fuentes: el mismo «Diario».

2. ALONSO, M. Les journaux espagnols durant la guerre d'Indépendance (1808-1815). 1981. $87 \mathrm{p}$.

3. ALONSO, M. La presse valencienne pendant l'époque napoléonienne (1808-1814). 1985. 332 p. (tesis)

Fuentes: los diarios cuya reseña aparece en el trabajo.

4. AZEMA, J., CARASSUS, A. la phénomène touristique de Majorque de 1945 à 1967. Mutations économiques et sociales. s.f. 400 p.

5. BALENCY, E. L'affaire de Carcagente (1727-1728)* 1974. 111 p.

Fuentes: AHN, Inquisición, leg. 520.

Pub.: El proceso de Carcaixent, 1727-28. Un estudio sobre la vida y la sociedad de

Carcaixent en el siglo XVIII.

Prolég de F. TORRES FOS y J.M. FERNANDEZ ROS. Carcaixent, 1982. 125 p.

6. BALENCY, E. Les violences en Andalousie. XVIe-XVIIe siècles. 1982. 306 p. (tesis).

Fuentes: $A H N$, Inquisición (Pleitos criminales), Consejos. AGS, Registro General del Sello, Cámara de Castilla (Perdones del Viernes Santo).

7. BERNARD, E., VALMARY, C. Une affaire à Ubeda. Crime et société au XVII siècle*. 1975. $151 \mathrm{p}$.

Fuentes: un pleito de la Inquisición.

8. BERNARD, O. Legislation royale et pouvoirs locaux. Analyse des lois de la Novisima Recopilación relatives aux communautés rurales. s.f. 61 p. 
9. BLANC, N.; LOPEZ, M.C. Moeurs et religion en Espagne au XVIIIe siècle. L'affaire de Teruel*. 1974. 88 p.

10. BLAZY, V. L'histoire de l'Espagne contemporaine (1936-1975) et le cinéma espagnol: pratiques discursives et idéologie. 1985. 243 p.

11. BRAULT-NOBLE, C. Inquisition, orthodoxie et ordre moral en Estrémadure à l'époque de Philippe II (de 1563 à 1575)*. 1976. 183 p.

12. BRAULT-NOBLE, C. Contribution à l'étude de la communauté marrane espagnole du bassin méditerranéen de la fin du XVe siècle jusqu'au XVIIe siècle, d'après les récits des voyageurs occidentaux. 1984. 418 p. (tesis).

13. BRUMONT, F. Briviesca: économie et société d'un bourg castillan au milieu du XVIe siècle. $1969.82 \mathrm{p}$.

Fuentes: AGS, Expedientes de Hacienda, leg. ${ }^{\circ}$ 60, exp. 37.

14. BRUMONT, F. La Bureba à la époque de Philippe II. Essai d'histoire rurale quantitative*. 1974. 161 p. más un volumen de cuadros, mapas y gráficos. (tesis).

Pub.: New-York: Arno Press, 1977.

Edición en castellano, ampliada y corregida: Campo y campesinos de Castilla la Vieja en tiempos de Felipe II. Madrid: Siglo XXI. 1984. 362 p.

15. CALMELS, A Deux ordonnances municipales en Vieille-Castille: Aranda de Duero, Mogrovejo y Tanarrio. Traduction et étude comparée. s.f.

16. CATHALA, P. Etude sur les communautés rurales en Vieille-Castille à l'époque moderne. 1983. 193 p.

Fuentes: $A H N$, Consejos: ordenazas municipales de Palacios de la Sierra, Gumiel de Hizán, Fuentelcesped, Villalaín, Villalobón y Calahorra de Boedo.

17. CERTIAT-BARRERE, M. Profil intellectuel et de carrière des candidats aux places de la maison de l'Infantado à la fin du XVIIIe siècle*. 1980. 180 p.

Fuentes: $A H N$, Osuna: relaciones de títulos y de méritos de dichos candidatos.

18. CHARLES, J.L. Nouvelles données sur les comptes d'exploitation en Castille à l'époque moderne: la granja de Cendrera (1630-1711)*. 1981. 142 p.

Fuentes: $A H N$, Clero, libros 1407 y 1425 (libros de cuentas de dicha granja sita en el término de Sotopalacios (Burgos) y perteneciente al monasterio de Ríoseco).

Biblio.: CHARLES, J.L. «Production céréalière, autoconsommation et marché: la grange cistercienne de Cendrera (1630-1711)». Congreso de Historia rural. Siglos XV al XIX. Madrid. 1984, pp. 809-826.

CHARLES, J.L. «La granje cistercienne de Cendrera. 1630-1711», in: L'explotation des grands domaines dans l'Espagne d'Ancien Régime (ouvrage collectif). Toulouse: CNRS 1985, pp. 69-101.

19. COMBET, M. Les structures agraires dans la région de Ciudad-Rodrigo au milieu du XVIIIe siècle*. 1972. 306 p. más 4 mapas.

Fuentes: Catastro de la Ensenada (Respuestas Generales del partido de Ciudad-Rodrigo).

20. CUBERO, J. Structures économiques et sociales de Avila au milieu du XVIe siècle*. 1968.

95 p. +7 cuadros + VIII p.

Fuentes: $A G S$, Expedientes de Hacienda, leg. ${ }^{\circ} 54$ (1557-61).

21. DELPECH, E. Gijón. Démographie, économie et société (1579-1588)* 1973. 132 p.

Fuentes: $A G S$, Expedientes de Hacienda, leg. ${ }^{\circ}$ 46, exp. 20.

22. ESCODA, D. Production et niveau de richesse dans une ville moyenne d'Andalousie: l'enquête d'Ubeda en 1583. 1982. $101 \mathrm{p}$.

Fuentes: $A G S$, Expedientes de Hacienda, leg. 190.

23. FERNANDEZ, B. Les relations de causes à Logroño de 1582 à 1635. 1981. 209 p. Fuentes: $A H N$, Inquisición, libros 834, 835, 836. 
24. FONTAINE, A. Vision de l'Espagne à travers les récits des voyageurs étrangers* 1973.198 p. + documentos.

25. GRENIER, S., IGLESIAS, F. Violences et luttes de classes à Andujar au XVIIe siècle*. 1976. $192 \mathrm{p}$.

Fuentes: dos pleitos de la Inquisición.

26. GUILHEM, C. Des femmes, l'inquisition et la foi (1617-1654)*. 1977. 155 p.

Fuentes: $A H N$, Inquisición, leg.os $85,114,115$ (tribunal de Toledo).

27. ISAAC-GEORGES, J.P. Le mouvement ouvrier catalan (nord et sud) de 1930 à 1936. 1980. $622+X X V I I I$ p. (tesis dirigida por el profesor R. TREMPE).

28. LASCAUX, M.; SOULIE, J. Oviedo et Tineo au milieu du XVIe siècle*. 1973. 111 p. (Trata también de las aldeas de estas dos villas).

Fuentes: $A G S$, Expedientes de Hacienda.

29. LEFRANC, M.G. Mémoire sur les blaphèmes et les blasphèmateurs dans le royaume de Valence aux XVIe et XVIIe siècles*. s.f. 105 p.

Fuentes: $A H N$, Inquisición, leg. ${ }^{\circ} 519$ (pleitos).

30. LORA FRECHOSO, I. Révolution et inaltérabilité dans l'Eglise espagnole après le concile Vatican II (1962-1976). 1977. 440 p. (tesis).

31. LUIS, J.P. Le clergé français émigré en Espagne pendant la Révolution (1791-1801). 1985. 213 p.

Fuentes: $A H N$, Estado, Leg. ${ }^{\circ}$ s 4003 y 4034.

32. MARC, M.J. Comportement et mentalité en Estrémadure à la fin du XVIe siècle (1589-1592) s.f. 181 p.

Fuentes: $A H N$, Inquisición, leg. ${ }^{\circ} 1988$.

33. MAS, R. Recherches sur l'exploitation agricole en Nouvelle-Castille au XVIIIe siécle*. s.f. $142 \mathrm{p}$.

Fuentes: $A H N$, Jesuitas (cuentas de la hacienda de Valdemoro).

Biblio:: MAS, R.; BRUMONT, F. «L'exploitation des Jésuites à Valdemoro (1699-1766)», in: L'exploitation des grands domaines dans l'Espagne d'Ancien Régime (ouvrage collectif). Toulouse: CNRS, 1985, pp. 161-175.

34. MONTEMAYOR, J. Economie et société dans quelques basses vallées de la «Montagne centrale» à la fin du XVIe siècle. (Partie orientale de la Junta de los Nueve Valles de las Asturias de Santillana)*. 1973. 112 p.

Fuentes: AGS, Expedientes de Hacienda, leg. ${ }^{\circ} 46$.

35. NDAMBA KABONGO, A. Les esclaves à Séville au début du XVIIe siècle. Approche de leurs origines et de leur condition à partir des Baptêmes, Mariages et Enterrements enregistrés de 1600 à 1621 dans les Paroisses sévillanes de Sainte Marie et Saint Ildefonse*. 1970. 139 p. + anejos + LXXXVIII p. (Incluye la lista nominativa de todos los esclavos así como las cifras anuales de bautismos, matrimonios y defunciones para ambas parroquias).

36. NDAMBA KABONGO, A. Les esclaves à Cordoue au début du XVIIe siècle (1600-1621). Provenance et condition sociale*. 1975.275 p. más documentos y bibliografía.

Fuentes: Archivos parroquiales y notariales.

37. PEYRE, D. Comportements et mentalités dans le royaume de Grenade au XVIe siècle*. 1977. $188 \mathrm{p}$.

Fuentes: $A H N$, Inquisición, leg. ${ }^{\circ} 1953$.

Biblio.: un capítulo en BENNASSAR, B. et. al.: L'inquisition espagnole, XVe-XIXe siècle. París: Hachette, 1979. 
38. POUZALGUES, B. L'inquisition face à la bigamie: le cas de Martín Escribano à Valence au XVIIe siècle*, s.f. 70 p.

39. RIGALDIES, F. La terre et les hommes du piémont de la Sierra de Guadarrama au XVIIIe siècle*. 1965. 96 p. + 10 gráficos y 2 mapas. (Abarca 65 pueblos de la actual provincia de Madrid en tres zonas: el Real de Manzanares, la tierra de Buitrago, las tierras del Jarama).

Fuentes: las Respuestas Generales del Catastro de la Ensenada.

40. SALINERO, G. Economie et société dune ville espagnole et de son territoire dans la seconde moitié du XVIe siècle: Alcántara. 1982.278 p.

Fuentes: $A G S$, Expedientes de Hacienda, Leg. ${ }^{\circ}$ s 33 y 34.

41. SERVAT, M. Saint-Jacques de Compostelle. Démographie, économie et société (1579-1588)*. 1973. $132 \mathrm{p}$.

Fuentes: AGS, Expedientes de Hacienda, leg. 160.

42. VARILHES, G. Le donativo de 1636 de la Vieille-Castille: la contribution d'une société. 1980. $117 \mathrm{p}$.

Fuentes: AGS, Contadurias Generales, leg. 3251. 
